\title{
Diffusion of cosmic rays in MHD turbulence
}

\author{
Siyao $\mathbf{X u}^{a * *}$ \\ ${ }^{a}$ Institute for Advanced Study, \\ 1 Einstein Drive, Princeton, NJ 08540, USA; Hubble Fellow \\ E-mail: sxu@ias.edu
}

We review some recent findings on diffusion of cosmic rays (CRs) in magnetohydrodynamic (MHD) turbulence obtained by adopting the numerically-tested model of MHD turbulence, including perpendicular superdiffusion of CRs, inefficient gyroresonant scattering by Alfvén and slow modes with scale-dependent turbulence anisotropy, resonance-broadened Transit Time Damping (TTD) interaction, and mirror diffusion. As the diffusion behavior of CRs strongly depends on the properties of MHD turbulence, theoretical modeling of CR diffusion, its numerical testing, and interpretation of CR-related observations require proper modeling of MHD turbulence.

$37^{\text {th }}$ International Cosmic Ray Conference (ICRC 2021)

July 12th - 23rd, 2021

Online - Berlin, Germany

\footnotetext{
${ }^{*}$ Presenter
} 


\section{Introduction}

Diffusion of cosmic rays (CRs) is an important physical process in space and astrophysical environments. It is important for probing the sources and chemical composition of CRs, studying the shock acceleration mechanisms, and understanding the roles of CRs in affecting star formation, galaxy evolution, feedback heating in galaxy clusters (e.g.,[1-6]).

The modeling of CR diffusion depends on the modeling of turbulent magnetic fields that they interact with. An ad hoc model of magnetohydrodynamic (MHD) waves with imposed Kolmogorov energy spectrum cannot describe the dynamics of turbulent magnetic fields. The 2D/slab superposition model of solar wind turbulence [7], although it contains a turbulence component of magnetic fields, cannot describe the scale-dependent anisotropy of MHD turbulence, because the important dynamical coupling between the turbulent motion perpendicular to the local magnetic field and the wave-like motion parallel to the local magnetic field is missing. The model of isotropic MHD turbulence is not applicable to anisotropic MHD turbulence when the effect of magnetic field is not negligible. The numerically-tested model of MHD turbulence that contains the physics on the dynamics of turbulent magnetic fields (e.g., [8-14]) is essential for studying CR diffusion in realistic astrophysical media. The theoretically predicted and numerically tested scale-dependent anisotropy of MHD turbulence is verified by solar wind observations (e.g., [15, 16]). The diffusion coefficient indicated by high-precision AMS-02 measurements of high-energy CRs also supports the numerically-tested model of MHD turbulence [17].

CR diffusion and associated acceleration strongly depends on the properties of MHD turbulence [18-27]. For instance, the scale-dependent anisotropy of Alfvén and slow modes causes inefficient gyroresonant scattering and Transit Time Damping (TTD) interaction [18, 19, 25, 28]. The superdiffusion of magnetic fields in Alfvénic turbulence results in the perpendicular superdiffusion of CRs on scales smaller than the driving scale of turbulence [23]. The perpendicular superdiffusion also affects the parallel diffusion of CRs along the turbulent magnetic field and introduces mirror diffusion of CRs [29]. These findings are important for theoretical modeling of CR diffusion. Furthermore, as the interstellar turbulence has a variety of turbulence regimes, realistic modeling of CR diffusion also requires information on turbulence properties, which can be obtained via statistical measurements of turbulence parameters (e.g., [30-32]).

\section{Interstellar magnetized turbulence and model for MHD turbulence}

The interstellar medium (ISM) is both turbulent and magnetized. The Big Power Law in the Sky shows a Kolmogorov spectrum of electron density fluctuations extending over 10 orders of magnitude in length scales [33-35]. This composite power-law spectrum is obtained from different measurements, including $\mathrm{H} \alpha$ emission at high Galactic latitudes and scattering measurements of nearby pulsars, and thus reflects the turbulence properties in the local ISM and in the warm ionized medium. In the multi-phase ISM, turbulence measurements with cold gas tracers reveal shallow density spectra (compared to the Kolmogorov one) in the cold neutral medium and molecular clouds $[36,37]$. Interstellar scattering and dispersion measures of distant pulsars also show shallow density spectra with excess of high-density structures toward smaller scales in the Galactic disk [38, 39]. The variety of turbulence in the multi-phase ISM can be further quantified by measuring turbulence 
parameters, e.g., Alfvénic and sonic Mach numbers, $M_{A}$ and $M_{S}$ (e.g., [30-32]). Mapping of $M_{A}$ and $M_{s}$ can provide detailed information on the magnetization and compressibility of the turbulent medium in different gas phases.

Depending on the values of $M_{A}=V_{L} / V_{A}$ and $M_{s}=V_{L} / c_{s}$, where $V_{L}$ is the injected turbulent speed at $L, L$ is the injection scale of turbulence and is of the order of $100 \mathrm{pc}$ in the ISM [40], $V_{A}$ is the Alfvén speed, and $c_{s}$ is the sound speed, turbulence can be in super-Alfvénic $\left(M_{A}>1\right)$, sub-Alfvénic $\left(M_{A}<1\right)$, super-sonic $\left(M_{S}>1\right)$, and sub-sonic $\left(M_{S}<1\right)$ regimes. Incompressible MHD turbulence was modeled by [8] and [9]. It was found that strong MHD turbulence with strong nonlinear interaction between oppositely moving wave packets [8] can be developed on scales smaller than $l_{A}=L M_{A}^{-3}$ in super-Alfvénic turbulence and $l_{\text {tran }}=L M_{A}^{2}$ in sub-Alfvénic turbulence [41]. Strong MHD turbulence has the scale-dependent anisotropy, with more elongated turbulent eddies along the turbulent energy cascade toward smaller scales, as tested by MHD turbulence simulations [10, 42]. Using the critical balance [8] between the turbulent eddy-turnover time in the direction perpendicular to the local magnetic field and the Alfvén-wave crossing time in the direction parallel to the local magnetic field, i.e.,

$$
\tau_{\text {tur }}=l_{\|} / V_{A},
$$

where the cascading rate is

$$
\tau_{\mathrm{tur}}^{-1}=v_{l} l_{\perp}^{-1}=V_{\mathrm{st}} L_{\mathrm{st}}^{-\frac{1}{3}} l_{\perp}^{-\frac{2}{3}},
$$

and $v_{l}$ is the turbulent speed at length scale $l$, the anisotropic scaling relation between the parallel scale $l_{\|}$and perpendicular scale $l_{\perp}$ of the turbulent eddy in strong MHD turbulence can be obtained,

$$
l_{\|}=\frac{V_{A}}{V_{\mathrm{st}}} L_{\mathrm{st}}^{\frac{1}{3}} l_{\perp}^{\frac{2}{3}}
$$

where [41]

$$
V_{\mathrm{st}}=V_{A}, \quad L_{\mathrm{st}}=l_{A}
$$

for super-Alfvénic turbulence, and

$$
V_{\mathrm{st}}=V_{L} M_{A}, \quad L_{\mathrm{st}}=l_{\text {tran }}
$$

for sub-Alfvénic turbulence.

Compressible MHD turbulence has been theoretically and numerically studied by, e.g., [11-13, 43-46], in both sub- and super-sonic MHD turbulence regimes. The decomposition of compressible MHD turbulence into Alfvén, slow, and fast modes [12, 43] allows one to separately study the properties of different turbulence modes and identify their roles in CR diffusion. The scaledependent anisotropy of incompressible MHD turbulence is found for Alfvén modes and slow modes in compressible MHD turbulence. While slow modes are passively mixed by Alfvén modes, fast modes have their own independent cascade and isotropic energy scaling [11, 12, 43]. These fundamental properties of turbulence modes have important effects on the pitch-angle scattering and spatial diffusion of CRs. The energy fractions of different turbulence modes depend on $M_{s}$ and $M_{A}$ [43]. Their measurements (see above) are important for realistic modeling of CR diffusion. 


\section{Perpendicular superdiffusion}

Perpendicular superdiffusion of CRs arises from the perpendicular superdiffusion of turbulent magnetic fields that are regulated by Alfvén modes. On scales smaller than $l_{A}$ in super-Alfvénic turbulence and $l_{\text {tran }}$ in sub-Alfvénic turbulence, similar to the Richardson dispersion of a pair of fluid particles in hydrodynamic turbulence [47], the separation between magnetic field lines also experiences accelerated growth as the field lines are separated by larger and larger turbulent eddies [23]. The perpendicular superdiffusion of turbulent magnetic fields has been tested by MHD turbulence simulations (e.g., [48, 49]). This superdiffusion of magnetic fields is enabled by turbulent reconnection of magnetic fields [9]. Otherwise magnetic fields can only have wave-like oscillations. The superdiffusion in super-Alfvénic turbulence is in the direction perpendicular to the magnetic field averaged over $l_{A}$ and in sub-Alfvénic turbulence is in the direction perpendicular to the global mean magnetic field.

CRs that travel along turbulent magnetic fields naturally have perpendicular superdiffusion. Their superdiffusion behavior depends on the relation between the scattering mean free path $\lambda_{\|}$and their traveling distance $|\delta \widetilde{x}|$ along the magnetic field line. When the scattering is inefficient with $\lambda_{\|}>|\delta \widetilde{x}|$, CRs have the same superdiffusion as turbulent magnetic fields, i.e., $\left\langle(\delta \widetilde{z})^{2}\right\rangle^{1 / 2} \propto|\delta \widetilde{x}|^{1.5}$, where $\left\langle(\delta \widetilde{z})^{2}\right\rangle^{1 / 2}$ is the rms separation between the trajectories of a pair of CRs, as shown by the test particle simulations in [21] (see Fig. 1(a)). When the scattering is efficient with $\lambda_{\|}<|\delta \widetilde{x}|$, the scaling of perpendicular superdiffusion of CRs deviates from that of turbulent magnetic fields, with $\left\langle(\delta \widetilde{z})^{2}\right\rangle^{1 / 2} \propto|\delta \widetilde{x}|^{0.75}$ (Fig. 1(b)). We stress that as the superdiffusion of CRs arises from the intrinsic superdiffusion of turbulent magnetic fields, for the numerical study of CR superdiffusion, it is important to use MHD turbulence simulations rather than ad hoc models of MHD turbulence.

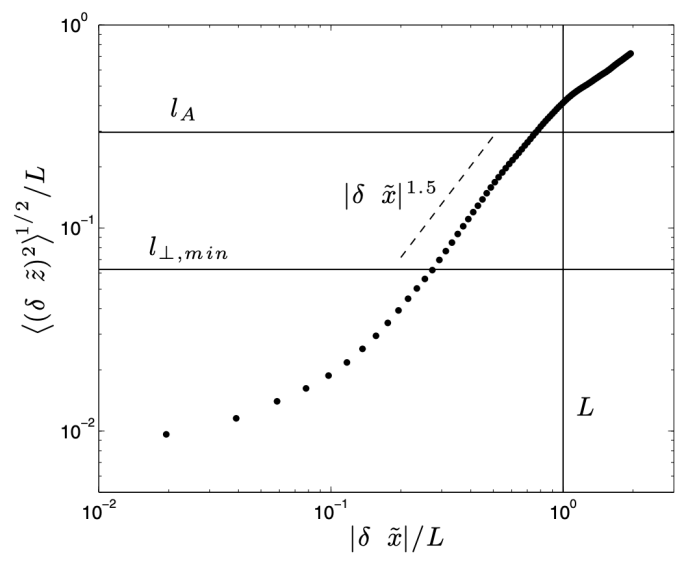

(a) $\lambda_{\|}>|\delta \tilde{x}|$

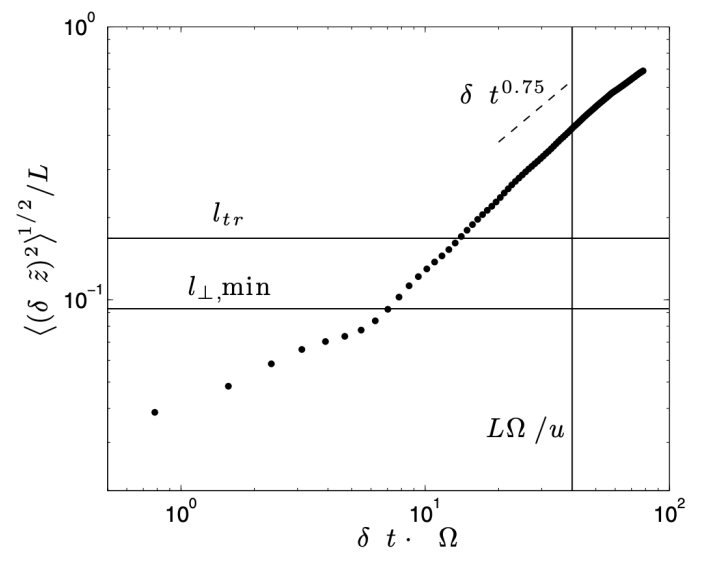

(b) $\lambda_{\|}<|\delta \tilde{x}|$

Figure 1: Perpendicular superdiffusion of CRs measured in test particle simulations. Note that $\delta t \propto|\delta \widetilde{x}|$ in (b). From [21].

In addition to $\mathrm{CR}$ perpendicular superdiffusion, the dynamics of turbulent magnetic fields also has important implications on resonance-broadened TTD interaction (see Section 5), reconnection acceleration of CRs [50], and mirror diffusion of CRs (see Section 6). 


\section{Gyroresonant scattering by MHD turbulence}

It was found by, e.g., [18, 19], that Alfvén modes are inefficient in scattering CRs due to the scale-dependent anisotropy. By using the anisotropic scaling of Alfvén modes in trans-Alfvénic turbulence $\left(M_{A}=1\right)$ and the resonance function in the quasilinear approximation

$$
R_{L}=\pi \delta\left(\omega_{k}-v_{\|} k_{\|}+\Omega\right),
$$

the pitch-angle diffusion coefficient for gyroresonant scattering by Alfvén modes is approximately [28]

$$
D_{\mu \mu, A} \approx \frac{2}{3} 8^{\frac{13}{2}} \exp (-8) \frac{\delta B_{A}^{2}}{B_{0}^{2}}\left(\frac{v}{L \Omega}\right)^{\frac{3}{2}} \frac{v}{L}\left(1-\mu^{2}\right)^{-\frac{1}{2}} \mu^{\frac{11}{2}},
$$

where $\omega_{k}$ is the wave frequency, $k_{\|}$is the parallel component of wavenumber, $v$ is the particle velocity, $\Omega$ is the gyrofrequency, $B_{0}$ is the strength of mean magnetic field, $\delta B_{A}$ is the magnetic perturbation induced by Alfvén modes at $L, \mu$ is the pitch-angle cosine, and $v_{\|}=v \mu$. The strong anisotropy in scattering by Alfvén modes arises from the scale-dependent anisotropy, which has a more significant effect at a smaller $\mu$.

Similar to Alfvén modes, slow modes also lead to inefficient gyroresonant scattering, with the pitch-angle diffusion coefficient [28]

$$
D_{\mu \mu, s} \approx \frac{2}{3} 8^{\frac{13}{2}} \exp (-8) \frac{\delta B_{s}^{2}}{B_{0}^{2}}\left(\frac{v}{L \Omega}\right)^{\frac{3}{2}} \frac{v}{L}\left(1-\mu^{2}\right)^{\frac{1}{2}} \mu^{\frac{7}{2}}=\frac{\delta B_{s}^{2}}{\delta B_{A}^{2}} \frac{1-\mu^{2}}{\mu^{2}} D_{\mu \mu, A},
$$

where $\delta B_{S}$ is the magnetic perturbation of slow modes at $L$. Fig. 2 displays the pitch-angle diffusion coefficients of TeV CRs for gyroresonant scattering by Alfvén and slow modes. Here $L=30 \mathrm{pc}$ and $\delta B_{A}=\delta B_{s}=B_{0}=3 \mu \mathrm{G}$ are assumed. We note that the above expressions do not apply to higher-energy CRs at a large $\mu$ due to the insignificant turbulence anisotropy on large scales in trans-Alfvénic turbulence.

Fast modes have independent energy cascade and isotropic energy scaling. The pitch-angle diffusion coefficient for gyroresonant scattering by fast modes at a large $\mu$ is approximately [28]

$$
D_{\mu \mu, f} \approx \frac{\pi}{56} \frac{\delta B_{f}^{2}}{B_{0}^{2}}\left(\frac{v}{L \Omega}\right)^{\frac{1}{2}} \Omega\left(1-\mu^{2}\right) \mu^{\frac{1}{2}},
$$

where $\delta B_{f}$ is the magnetic perturbation of fast modes at $L$. Due to the isotropic scaling, the scattering by fast modes is efficient and anisotropy in scattering is weak. We note that $D_{\mu \mu, f}$ decreases with increasing CR energy, while $D_{\mu \mu, A}$ and $D_{\mu \mu, s}$ increase with CR energy due to the weaker turbulence anisotropy on larger length scales.

\section{Resonance-broadened TTD of CRs}

TTD is the magnetic analog of Landau damping. Via the TTD resonant interaction of CRs with magnetic compressions, CRs undergo the second order Fermi acceleration [51]. The stochastic acceleration causes the stochastic increase of $\mu$. This effective "pitch angle diffusion" should be distinguished from the pitch-angle diffusion due to gyroresonant scattering in Section 4. 


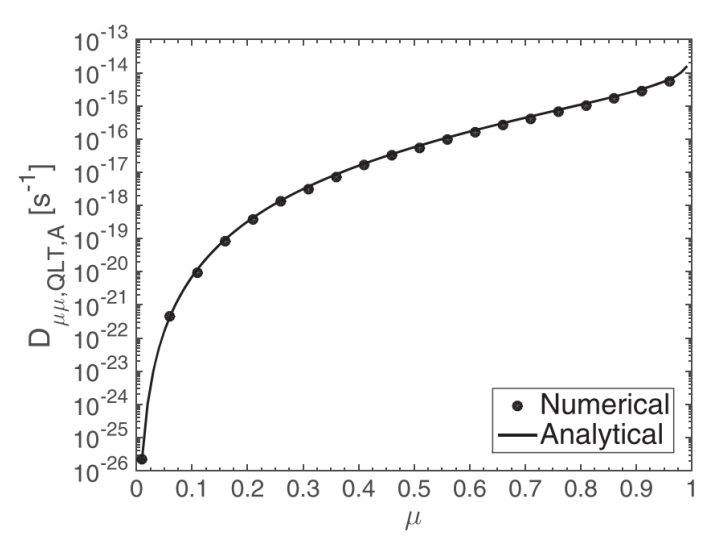

(a) Alfvén modes

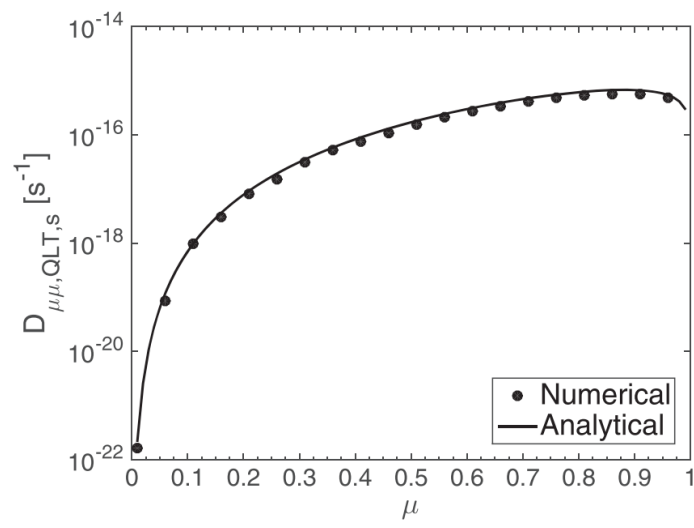

(b) Slow modes

Figure 2: Pitch-angle diffusion coefficients of TeV CRs for gyroresonant scattering by Alfvén and slow modes. "Numerical" means the results calculated by numerically integrating the magnetic energy spectrum (see [28]). "Analytical" means the approximate analytical expressions given by Eq. (7) and Eq. (8). From [28].

The linear resonance function for TTD is

$$
R_{L}=\pi \delta\left(\omega_{k}-v_{\|} k_{\|}\right)
$$

It means that the TTD interaction happens when $v_{\|}$matches the wave parallel phase speed $\omega_{k} / k_{\|}$. With the linear resonance, the pitch-angle diffusion coefficient for TTD with slow modes is [25]

$$
D_{\mu \mu, s L, T}=\frac{\pi^{2}}{2} \frac{C_{s}}{B_{0}^{2}} L^{-\frac{2}{3}} \ln \left(\frac{L}{l_{\perp, \min }}\right) v^{2}\left(1-\mu^{2}\right)^{2} \delta\left(v_{\|}-V_{\mathrm{ph}}\right)
$$

where

$$
C_{s}=\frac{1}{6 \pi} \delta B_{s}^{2} L^{-\frac{1}{3}},
$$

$V_{\mathrm{ph}}$ is the wave phase speed, and $l_{\perp \text {,min }}$ is determined by the larger one between the gyroradius $r_{g}$ and the dissipation scale $l_{d}$ of magnetic fluctuations. Only particles that exactly match the discrete resonance condition can undergo TTD interaction. The pitch-angle diffusion coefficient for TTD with fast modes is [25]

$$
D_{\mu \mu, f L, T}=2 H^{*} \pi^{2} \frac{C_{f}}{B_{0}^{2}}\left(l_{\min }^{-\frac{1}{2}}-L^{-\frac{1}{2}}\right) v \frac{\left(1-\mu^{2}\right)^{2}}{\mu}\left(\frac{V_{\mathrm{ph}}}{v_{\|}}\right)^{2}\left[1-\left(\frac{V_{\mathrm{ph}}}{v_{\|}}\right)^{2}\right],
$$

where

$$
\begin{gathered}
C_{f}=\frac{1}{16 \pi} \delta B_{f}^{2} L^{-\frac{1}{2}}, \\
H^{*}=H\left(1-\frac{V_{\mathrm{ph}}}{v_{\|}}\right) H\left(\frac{V_{\mathrm{ph}}}{v_{\|}}\right),
\end{gathered}
$$

$H$ is the Heaviside step function, and $l_{\min }$ is determined by the larger one between $r_{g}$ and $l_{d}$ of fast modes. The particles that satisfy $v_{\|} \geq V_{\text {ph }}$ can have TTD interaction with fast modes. 
In MHD turbulence, both the magnetic fluctuations induced by compressible turbulence modes $[52,53]$ and the limited lifetime of turbulent eddies [20] can cause broadening of quasi-linear resonances. By taking into account both broadening effects, the general form of the broadened resonance function is [25],

$$
R_{B}=\frac{\sqrt{2 \pi}}{2\left(\Delta v_{\|} k_{\|}+\omega_{\text {tur }}\right)} \exp \left[-\frac{\left(\omega_{k}-v_{\|} k_{\|}\right)^{2}}{2\left(\Delta v_{\|} k_{\|}+\omega_{\text {tur }}\right)^{2}}\right],
$$

where $\Delta v_{\|}$is the variation in $v_{\|}$caused by parallel magnetic perturbation, and $\omega_{\text {tur }}=\tau_{\text {tur }}^{-1}$. For CRs with $\Delta v_{\|} k_{\|} \gg \omega_{\text {tur }}$, the broadening is dominated by magnetic fluctuations.

With resonance broadening, the pitch-angle diffusion coefficient for TTD with slow modes is [25]

$$
D_{\mu \mu, s B, T} \approx \frac{\sqrt{2}}{4} \pi^{\frac{3}{2}} \frac{C_{s}}{B_{0}^{2}}\left(\Delta v_{\|}+V_{A}\right)^{-1} L^{-\frac{2}{3}} \ln \left(\frac{L}{l_{\perp, \min }}\right) v^{2}\left(1-\mu^{2}\right)^{2} \exp \left[-\frac{\left(V_{\mathrm{ph}}-v_{\|}\right)^{2}}{2\left(\Delta v_{\|}+V_{A}\right)^{2}}\right] .
$$

With the broadened resonance, particles with a broad range of $v$ can have TTD interaction with slow modes. For high-energy CRs, the broadening is dominated by the effect of magnetic fluctuations, and the above expression can be simplified,

$$
D_{\mu \mu, S B, T} \approx \frac{\sqrt{2}}{4} \pi^{\frac{3}{2}} \frac{C_{S}}{B_{0}^{2}}\left(\frac{\left\langle\delta B_{\|}^{2}\right\rangle}{B_{0}^{2}}\right)^{-\frac{1}{4}} L^{-\frac{2}{3}} \ln \left(\frac{L}{l_{\perp, \min }}\right) v\left(1-\mu^{2}\right)^{\frac{3}{2}} \exp \left[-\frac{v_{\|}^{2}}{2 \Delta v_{\|}^{2}}\right] .
$$

At a small $\mu$, it approximately remains constant. The pitch-angle diffusion coefficient for TTD of high-energy particles with fast modes is [25]

$$
D_{\mu \mu, f B, T} \approx \frac{\sqrt{2}}{4} \pi^{\frac{3}{2}} \frac{C_{f}}{B_{0}^{2}}\left(\frac{\left\langle\delta B_{\|}^{2}\right\rangle}{B_{0}^{2}}\right)^{-\frac{1}{4}}\left(l_{\min }^{-\frac{1}{2}}-L^{-\frac{1}{2}}\right) v\left(1-\mu^{2}\right)^{\frac{3}{2}} \exp \left[-\frac{v_{\|}^{2}}{2 \Delta v_{\|}^{2}}\right] .
$$

Compared with the case with linear resonance, the broadened resonance enables efficient TTD interaction for high-energy CRs. As an example, Fig. 3 displays the pitch-angle diffusion coefficients of TeV CRs for resonance-broadened TTD with slow and fast modes in an environment similar to the interstellar warm ionized medium. In this illustration, it is assumed that slow and fast modes contain comparable injected turbulent energies. Except for large $\mu$, resonance-broadened TTD interaction of high-energy particles is effective.

The comparison between the pitch-angle diffusion coefficients of high-energy particles for resonance-broadened TTD with slow and fast modes depends on plasma $\beta$ [25],

$$
\frac{D_{\mu \mu, S B, T}}{D_{\mu \mu, F B, T}}=\frac{4}{3} \frac{V_{L s}^{2}}{V_{L f}^{2}} \frac{\ln \left(\frac{L}{l_{\perp, \mathrm{min}, \mathrm{S}}}\right)}{\sqrt{\frac{L}{l_{\min , \mathrm{f}}}}-1} \beta,
$$

where $V_{L s}$ and $V_{L f}$ are the injected turbulent speeds of slow and fast modes. By assuming $V_{L s} \sim V_{L f}$ and $r_{g}$ larger than the dissipation scales of both slow and fast modes, Fig. 4 shows the relative importance between slow and fast modes in TTD as a function of $\beta$ and CR energy $E_{\mathrm{CR}}$, where the parameters $L=30 \mathrm{pc}$ and $B_{0}=3 \mu \mathrm{G}$ are adopted. As indicated by Eq. (20), 


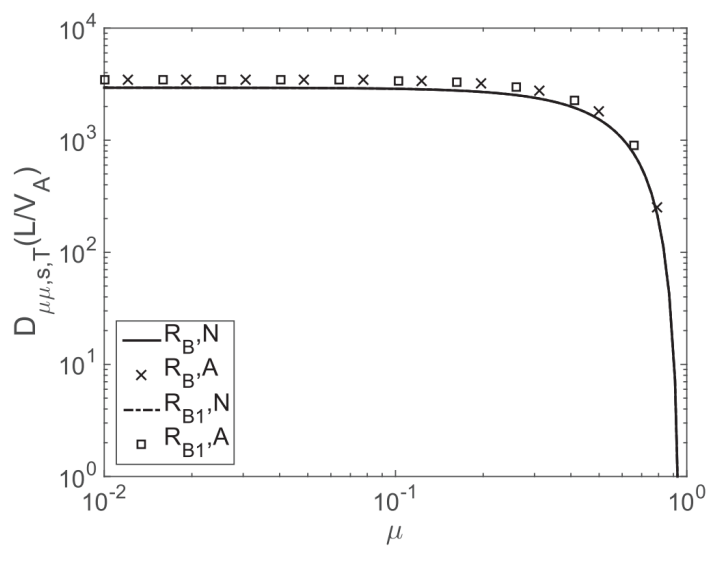

(a) Slow modes

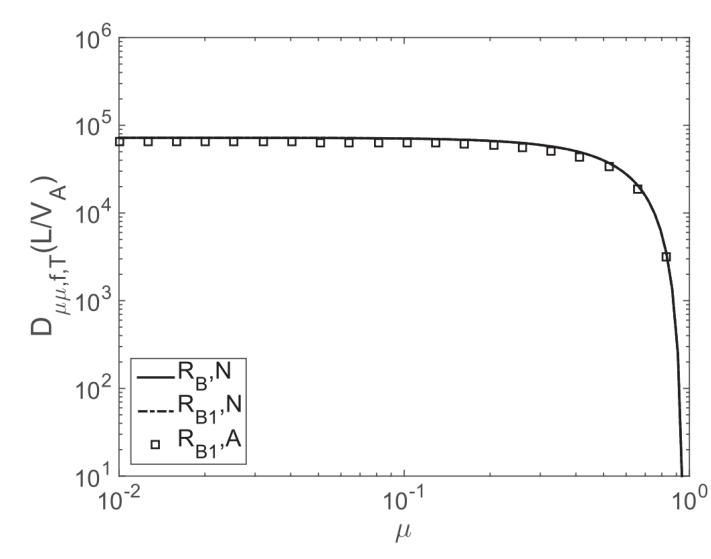

(b) Fast modes

Figure 3: Pitch-angle diffusion coefficients (normalized by $V_{A} / L$ ) of TeV CRs for resonance-broadened TTD with slow and fast modes in the warm ionized medium. "N" corresponds to the result calculated with numerical integration. "A" corresponds to the analytical estimation as given in the text. $R_{B}$ and $R_{B} 1$ correspond to the general form of broadened resonance function and that dominated by the effect of magnetic fluctuations. From [25].

slow modes make more significant contribution to TTD at a large $\beta$. For low-energy CRs, due to the turbulence anisotropy and thus small magnetic fluctuation parallel to the magnetic field of slow modes at small scales, TTD with slow modes is inefficient. Note that if there is $V_{L s} \gg V_{L f}$ for the driven turbulence, slow modes can play a more important role in TTD than the case illustrated in Fig. 4.

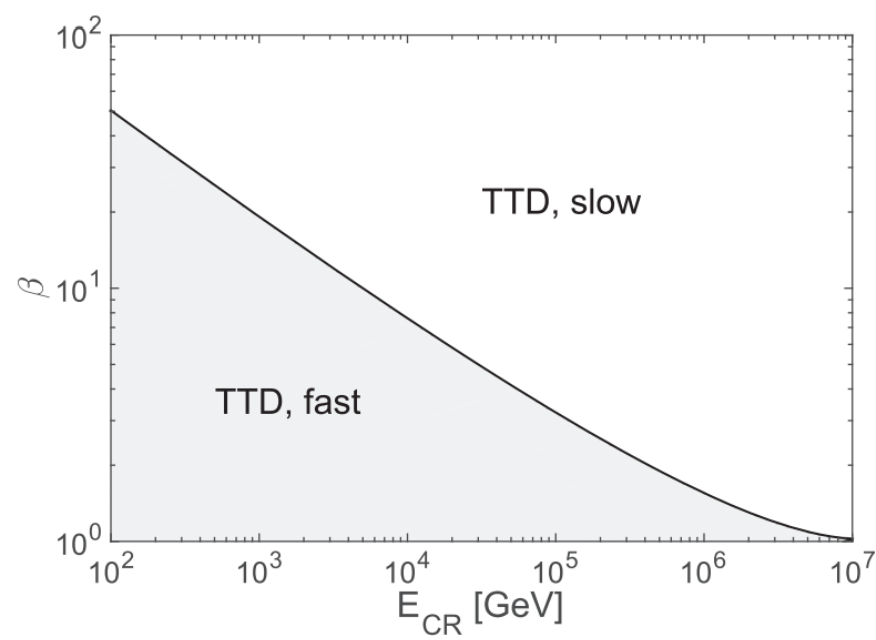

Figure 4: The relative importance between slow and fast modes in resonance-broadened TTD as a function of $\beta$ and $E_{\mathrm{CR}}$. The shaded region shows the parameter space where fast modes dominate TTD. From [25]. 


\section{Mirror diffusion}

In MHD turbulence, slow and fast modes generate magnetic compressions, which act as magnetic mirrors. Particles with conserved magnetic moment, $p_{\perp}^{2} / B$, where $p_{\perp}$ is the perpendicular component of particle momentum, can undergo mirror reflection if $\mu$ is smaller than

$$
\mu_{l c}=\sqrt{\frac{\delta b}{B_{0}+\delta b}},
$$

where the magnetic perturbation $\delta b$ is determined by that at wavenumber $k$, i.e., $b_{k}$, for slow and fast modes with a spectrum of magnetic fluctuations. The magnetic mirroring effect leads to the trapping of particle between the mirror points in a static magnetic bottle (e.g., [54, 55]). However, in MHD turbulence with the superdiffusion of turbulent magnetic fields induced by Alfvén modes (see Section 3), particles that interact with magnetic mirrors also undergo superdiffusion in the direction perpendicular to magnetic field. As a result, particles cannot be confined within the same "magnetic bottle", but stochastically encounter the mirror points of different "magnetic bottles" when they propagate super-diffusively following the field lines regulated by Alfvén modes (see Fig. 5). The magnetic mirroring causes diffusion rather than trapping of CRs along the magnetic field line, which is termed "mirror diffusion" [29].

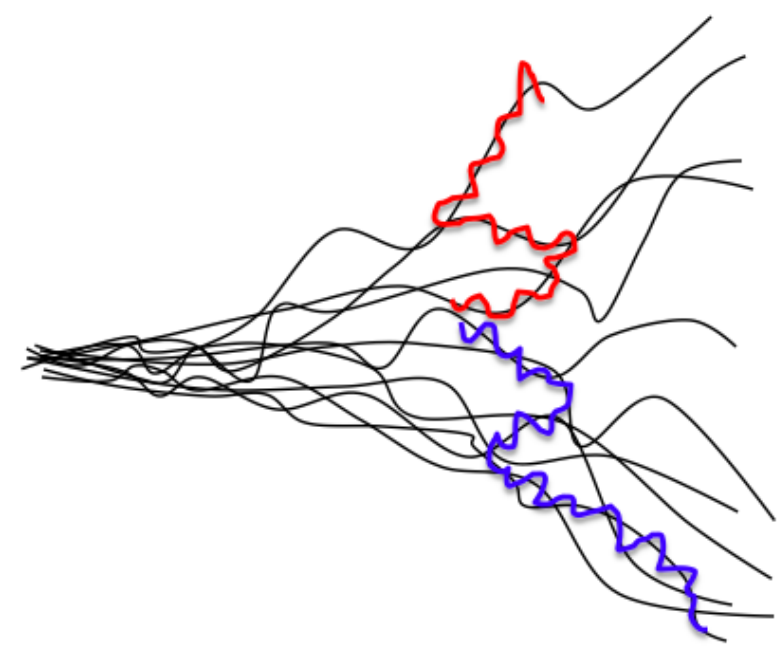

Figure 5: CRs undergo both perpendicular superdiffusion and parallel mirror diffusion in MHD turbulence. Red and blues lines represent the trajectories of CRs with small initial separation. Thin black lines represent turbulent magnetic fields with both perpendicular superdiffusion induced by Alfvén modes and magnetic compressions induced by slow and fast modes. From [29].

In the case when fast modes dominate both mirroring and gyroresonant scattering (Section 4), the parallel diffusion coefficient of particles that bounce with magnetic mirrors, i.e., bouncing particles, is [29]

$$
D_{\|, f, b}=\frac{1}{10} \nu L\left(\frac{\delta B_{f}}{B_{0}}\right)^{-4} \mu_{c}^{10},
$$

where

$$
\mu_{c} \approx\left[\frac{14}{\pi} \frac{\delta B_{f}^{2}}{B_{0}^{2}}\left(\frac{v}{L \Omega}\right)^{\frac{1}{2}}\right]^{\frac{2}{11}}
$$


is the critical $\mu$ at the balance between mirroring and scattering. In the presence of mirroring, the range of $\mu$ for pitch-angle scattering is limited to $\left[\mu_{c}, 1\right]$. Accordingly, the parallel diffusion coefficient of the particles that do not bounce with magnetic mirrors, i.e., non-bouncing particles, becomes [28]

$$
D_{\|, f, n b}=\frac{v^{2}}{4} \int_{\mu_{c}}^{1} d \mu \frac{\left(1-\mu^{2}\right)^{2}}{D_{\mu \mu, f}} \approx \frac{28}{5 \pi} \frac{B_{0}^{2}}{\delta B_{f}^{2}}\left(\frac{v}{L \Omega}\right)^{-\frac{1}{2}} \frac{v^{2}}{\Omega}\left[4-\sqrt{\mu_{c}}\left(5-\mu_{c}^{2}\right)\right],
$$

where $D_{\mu \mu, f}$ is given by Eq. (9). Fig. 6 presents the parallel diffusion coefficients of bouncing and non-bouncing CRs that interact with fast modes, where the parameters $L=30 \mathrm{pc}$ and $\delta B_{f}=B_{0}=$ $3 \mu \mathrm{G}$ are assumed. It shows that $D_{\|, f, n b}$ of non-bouncing particles in the presence of mirroring has the energy scaling shallower than $E_{\mathrm{CR}}^{0.5}$ and is close to $E_{\mathrm{CR}}^{1 / 3}$ for most energies. The mirroring effect leads to slower diffusion of bouncing particles than that of non-bouncing particles, and the energy scaling of $D_{\|, f, b}$ is steeper than $D_{\|, f, n b}$.

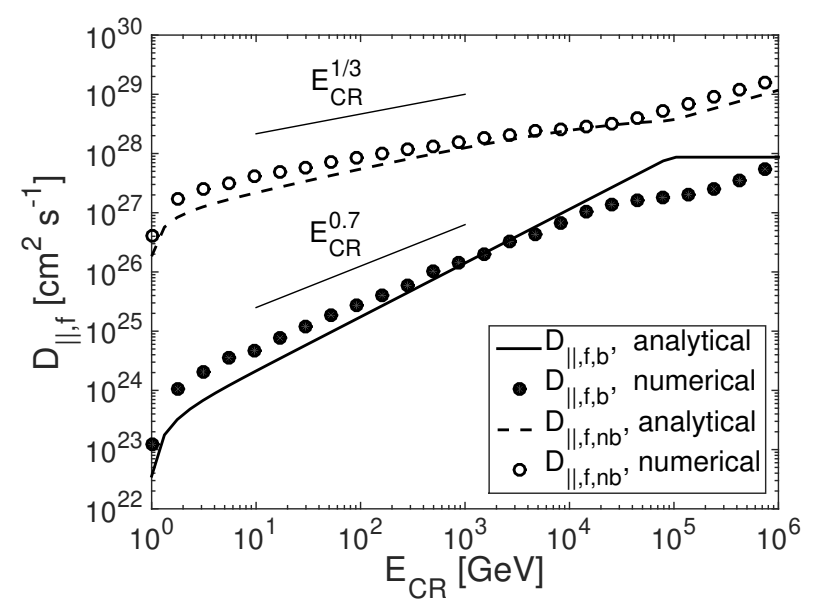

Figure 6: Parallel diffusion coefficients of bouncing and non-bouncing CRs when fast modes dominate both mirroring and scattering. "Numerical" means the result calculated with numerical integration, and "analytical" means the analytical approximation given in the text. From [29].

Fig. 7 further illustrates the parallel diffusion of CRs in different cases with varying energy fractions of fast modes in trans-Alfvénic turbulence. The energy fraction of slow modes is fixed with $\delta B_{s} / B_{0}=0.5$, and the pitch angle distribution is assumed to be isotropic. In the limit case of incompressible MHD turbulence in Fig. 7(a), the scattering by anisotropic Alfvén and pseudoAlfvén modes is inefficient. The corresponding parallel diffusion coefficient of non-bouncing particles $D_{\|, n b}$ is large and decreases with increasing $E_{\mathrm{CR}}$ because of the weaker turbulence anisotropy at larger length scales (Section 4). With the increase of energy fraction of fast modes, more efficient scattering leads to smaller $D_{\|, n b}$. For bouncing particles, pseudo-Alfvén/slow modes dominate the mirroring effect in Figs. 7(a)-7(c) when the energy fraction of fast modes is small. In Fig. $7\left(\right.$ d), fast modes dominate both scattering and mirroring. $D_{\|, \text {tot }}$ is the average parallel diffusion coefficient of CRs on scales much larger than the mean free paths of both bouncing and non-bouncing particles. With $D_{\|, n b}>D_{\|, b}, D_{\|, \text {tot }}$ is determined by the diffusion of nonbouncing particles. These examples show that the diffusion of CRs depends on the properties 
of MHD turbulence and is thus not homogeneous. In the multi-phase ISM with a much larger variety of turbulence regimes, the detailed energy fractions of different turbulence modes should be determined by measuring turbulence parameters $M_{S}$ and $M_{A}$ (see Section 2).

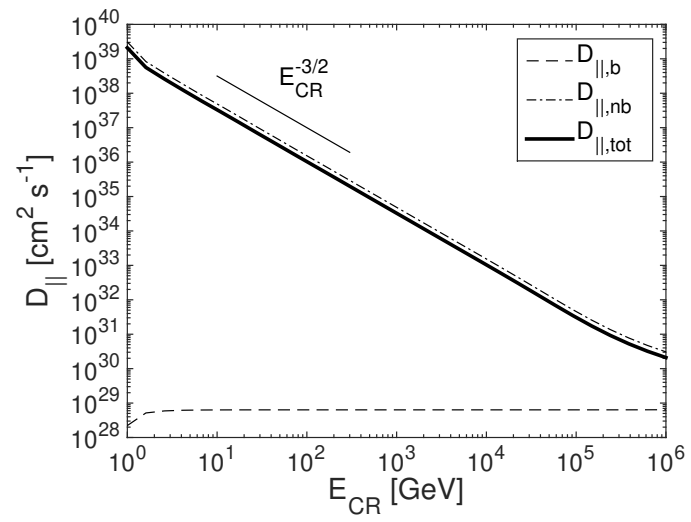

(a) $\delta B_{f} / B_{0}=0$

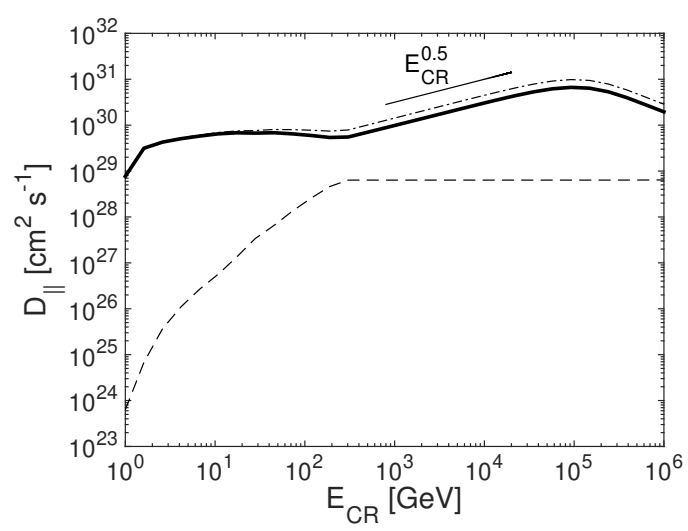

(c) $\delta B_{f} / B_{0}=0.1$

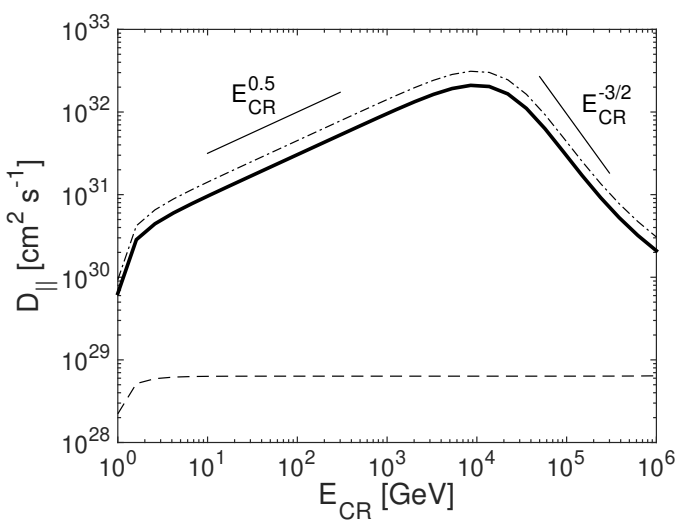

(b) $\delta B_{f} / B_{0}=0.01$

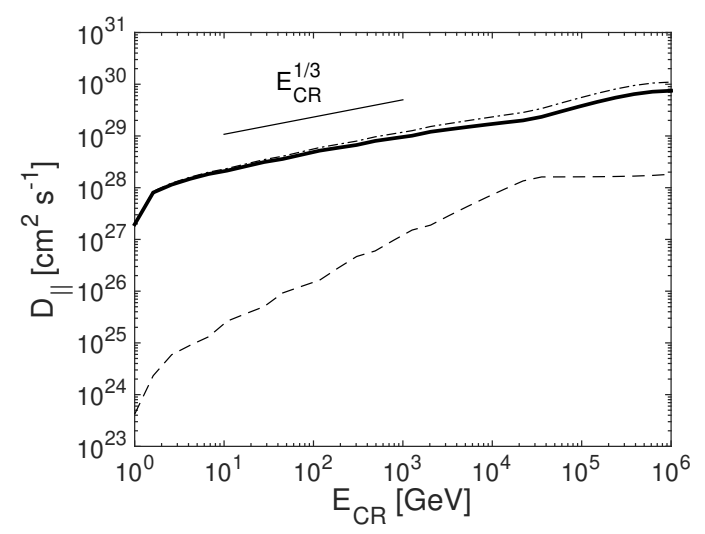

(d) $\delta B_{f} / B_{0}=0.5$

Figure 7: Parallel diffusion coefficients of bouncing particles $D_{\|, b}$, non-bouncing particles $D_{\|, n b}$, and their average value $D_{\|, \text {tot }}$ on scales much larger than the particle mean free paths in MHD turbulence with varying energy fractions of fast modes. From [29].

The slow diffusion of bouncing CRs in the vicinity of CR sources can have important implications on the diffusion of high-energy electrons and positrons around pulsar wind nebulae (e.g., [56]). The steep energy scaling of the diffusion coefficient of bouncing CRs can also be important for explaining the high-energy gamma-ray spectra of middle-aged supernova remnants [57].

\section{Summary}

(i) CR diffusion is sensitive to the properties of MHD turbulence that CRs interact with. A proper description of CR diffusion behavior requires a numerically tested model of MHD turbulence. In the multi-phase ISM with a large variety of turbulence regimes, based on the theoretical 
understanding on the relation of CR diffusion to MHD turbulence, observationally measuring turbulence parameters $M_{S}$ and $M_{A}$ that characterize the properties of MHD turbulence is necessary for realistic modeling of CR diffusion.

(ii) The perpendicular superdiffusion of CRs arises from the intrinsic perpendicular superdiffusion of magnetic fields in Alfvénic turbulence. This superdiffusion behavior can only be studied when using the numerically tested model of MHD turbulence that contains the physics of nonlinear wave interaction and turbulent energy cascade. Turbulent reconnection of magnetic fields naturally exists in this model, which allows the turbulent motion of magnetic fields. Without turbulent reconnection, field lines can only have wave-like oscillations due to the restoring magnetic tension force. As tested by test particle simulations, the perpendicular superdiffusion of CRs persists in the presence of diffusion of CRs along the turbulent magnetic field. On the one hand, the parallel diffusion can modify the scaling of superdiffusion if the parallel mean free path is sufficiently small. On the other hand, the perpendicular superdiffusion also induces the parallel mirror diffusion of particles in addition to the parallel diffusion associated with scattering.

(iii) For gyroresonant scattering, due to the scale-dependent anisotropy of Alfvén and slow modes, they are inefficient in scattering CRs especially toward low CR energies and small $\mu$.

(iv) TTD is a stochastic acceleration mechanism. The TTD acceleration causes stochastic increase of $\mu$. When considering the resonance broadening effect coming from both compressible magnetic fluctuations and limited life time of turbulent eddies, both low and high-energy particles can undergo TTD interaction. TTD with slow modes is relatively inefficient for low-energy CRs due to the turbulence anisotropy, but it becomes more efficient toward high CR energies and large plasma $\beta$.

(v) Because of the CR perpendicular superdiffusion, the magnetic mirroring effect causes diffusion of CRs along the turbulent magnetic field, instead of trapping of CRs. The CRs that are subject to mirror reflection have slow mirror diffusion. For CRs with small pitch angles that are not subject to mirror reflection, their parallel diffusion due to scattering and the energy scaling of diffusion coefficient are also affected in the presence of magnetic mirrors in MHD turbulence. The slow mirror diffusion has important implications on CR diffusion in the vicinity of CR sources.

\section{Acknowledgments}

S.X. acknowledges the support for this work provided by NASA through the NASA Hubble Fellowship grant \# HST-HF2-51473.001-A awarded by the Space Telescope Science Institute, which is operated by the Association of Universities for Research in Astronomy, Incorporated, under NASA contract NAS5-26555.

\section{References}

[1] J.R. Jokipii, Cosmic-Ray Propagation. I. Charged Particles in a Random Magnetic Field, ApJ 146 (1966) 480.

[2] R. Schlickeiser, Cosmic-ray transport and acceleration, ApJS 90 (1994) 929. 
[3] G. Brunetti and A. Lazarian, Compressible turbulence in galaxy clusters: physics and stochastic particle re-acceleration, MNRAS 378 (2007) 245 [arXiv:astro-ph/0703591].

[4] F. Holguin, M. Ruszkowski, A. Lazarian, R. Farber and H.Y.K. Yang, Role of cosmic-ray streaming and turbulent damping in driving galactic winds, MNRAS 490 (2019) 1271 [1807.05494].

[5] M.R. Krumholz, R.M. Crocker, S. Xu, A. Lazarian, M.T. Rosevear and J. Bedwell-Wilson, Cosmic ray transport in starburst galaxies, MNRAS 493 (2020) 2817 [1911.09774].

[6] E. Amato and S. Casanova, On particle acceleration and transport in plasmas in the Galaxy: theory and observations, Journal of Plasma Physics 87 (2021) 845870101.

[7] W.H. Matthaeus, M.L. Goldstein and D.A. Roberts, Evidence for the presence of quasi-two-dimensional nearly incompressible fluctuations in the solar wind, JGR 95 (1990) 20673.

[8] P. Goldreich and S. Sridhar, Toward a theory of interstellar turbulence. 2: Strong alfvenic turbulence, ApJ 438 (1995) 763.

[9] A. Lazarian and E.T. Vishniac, Reconnection in a Weakly Stochastic Field, ApJ 517 (1999) 700 [arXiv: astro-ph/9811037].

[10] J. Cho and E.T. Vishniac, The Anisotropy of Magnetohydrodynamic Alfvénic Turbulence, ApJ 539 (2000) 273 [arXiv: astro-ph/0003403].

[11] Y. Lithwick and P. Goldreich, Compressible Magnetohydrodynamic Turbulence in Interstellar Plasmas, ApJ 562 (2001) 279 [arXiv:astro-ph/0106425].

[12] J. Cho and A. Lazarian, Compressible Sub-Alfvénic MHD Turbulence in Low- $\beta$ Plasmas, Physical Review Letters 88 (2002) 245001 [arXiv: astro-ph/0205282].

[13] G. Kowal and A. Lazarian, Velocity Field of Compressible Magnetohydrodynamic Turbulence: Wavelet Decomposition and Mode Scalings, ApJ 720 (2010) 742 [1003. 3697].

[14] A. Beresnyak, Spectra of Strong Magnetohydrodynamic Turbulence from High-resolution Simulations, ApJ 784 (2014) L20 [1401 .4177].

[15] Q.Y. Luo and D.J. Wu, Observations of Anisotropic Scaling of Solar Wind Turbulence, ApJ 714 (2010) L138.

[16] M.A. Forman, R.T. Wicks and T.S. Horbury, Detailed Fit of "Critical Balance" Theory to Solar Wind Turbulence Measurements, ApJ 733 (2011) 76.

[17] O. Fornieri, D. Gaggero, S.S. Cerri, P. De La Torre Luque and S. Gabici, The theory of cosmic ray scattering on pre-existing MHD modes meets data, MNRAS 502 (2021) 5821 [2011.09197]. 
[18] B.D.G. Chandran, Scattering of Energetic Particles by Anisotropic Magnetohydrodynamic Turbulence with a Goldreich-Sridhar Power Spectrum, Physical Review Letters 85 (2000) 4656 [astro-ph/0008498].

[19] H. Yan and A. Lazarian, Scattering of Cosmic Rays by Magnetohydrodynamic Interstellar Turbulence, Physical Review Letters 89 (2002) B1102+ [arXiv: astro-ph/0205285].

[20] J.W. Lynn, I.J. Parrish, E. Quataert and B.D.G. Chandran, Resonance Broadening and Heating of Charged Particles in Magnetohydrodynamic Turbulence, ApJ 758 (2012) 78 [1204.0155].

[21] S. Xu and H. Yan, Cosmic-Ray Parallel and Perpendicular Transport in Turbulent Magnetic Fields, ApJ 779 (2013) 140 [1307.1346].

[22] G. Brunetti and A. Lazarian, Particle reacceleration by compressible turbulence in galaxy clusters: effects of a reduced mean free path, MNRAS 412 (2011) 817 [1011.1198].

[23] A. Lazarian and H. Yan, Superdiffusion of Cosmic Rays: Implications for Cosmic Ray Acceleration, ApJ 784 (2014) 38 [1308.3244].

[24] V. López-Barquero, R. Farber, S. Xu, P. Desiati and A. Lazarian, Cosmic-Ray Small-scale Anisotropies and Local Turbulent Magnetic Fields, ApJ 830 (2016) 19 [1509.00892].

[25] S. Xu and A. Lazarian, Resonance-broadened Transit Time Damping of Particles in MHD Turbulence, ApJ 868 (2018) 36 [1810.07726].

[26] S. Xu, N. Klingler, O. Kargaltsev and B. Zhang, On the Broadband Synchrotron Spectra of Pulsar Wind Nebulae, ApJ 872 (2019) 10 [1812 . 10827].

[27] C. Demidem, M. Lemoine and F. Casse, Particle acceleration in relativistic turbulence: A theoretical appraisal, Phys. Rev. D 102 (2020) 023003 [1909. 12885].

[28] S. Xu and A. Lazarian, Trapping of Cosmic Rays in MHD Turbulence, ApJ 894 (2020) 63 [2003.12593].

[29] A. Lazarian and S. Xu, Diffusion of cosmic rays in MHD turbulence with magnetic mirrors, arXiv:2106.08362 (2021) [2106.08362].

[30] A. Lazarian, K.H. Yuen, K.W. Ho, J. Chen, V. Lazarian, Z. Lu et al., Distribution of Velocity Gradient Orientations: Mapping Magnetization with the Velocity Gradient Technique, ApJ 865 (2018) 46 [1802 . 02984].

[31] K.H. Yuen and A. Lazarian, Advancing the Velocity Gradient Technique: Using Gradient Amplitudes and Handling Thermal Broadening, ApJ 898 (2020) 65.

[32] S. Xu and Y. Hu, Measuring Magnetization with Rotation Measures and Velocity Centroids in Supersonic MHD Turbulence, ApJ 910 (2021) 88 [2102.05647]. 
[33] J.W. Armstrong, B.J. Rickett and S.R. Spangler, Electron density power spectrum in the local interstellar medium, ApJ 443 (1995) 209.

[34] A. Chepurnov and A. Lazarian, Extending the Big Power Law in the Sky with Turbulence Spectra from Wisconsin Ho Mapper Data, ApJ 710 (2010) 853 [0905.4413].

[35] K.H. Lee and L.C. Lee, Turbulence Spectra of Electron Density and Magnetic Field Fluctuations in the Local Interstellar Medium, ApJ 904 (2020) 66.

[36] A. Lazarian, Obtaining Spectra of Turbulent Velocity from Observations, Space Science Reviews 143 (2009) 357 [0811. 0839].

[37] P. Hennebelle and E. Falgarone, Turbulent molecular clouds, AAPR 20 (2012) 55 [1211.0637].

[38] S. Xu and B. Zhang, Scatter Broadening of Pulsars and Implications on the Interstellar Medium Turbulence, ApJ 835 (2017) 2 [1610.03011].

[39] S. Xu and B. Zhang, Nonuniversal Interstellar Density Spectra Probed by Pulsars, ApJ 905 (2020) 159 [2011.01244].

[40] A. Chepurnov, A. Lazarian, S. Stanimirović, C. Heiles and J.E.G. Peek, Velocity Spectrum for H I at High Latitudes, ApJ 714 (2010) 1398 [astro-ph/0611462].

[41] A. Lazarian, Enhancement and Suppression of Heat Transfer by MHD Turbulence, ApJ 645 (2006) L25 [arXiv: astro-ph/0608045].

[42] J. Cho, A. Lazarian and E.T. Vishniac, Simulations of Magnetohydrodynamic Turbulence in a Strongly Magnetized Medium, ApJ 564 (2002) 291 [arXiv: astro-ph/0105235].

[43] J. Cho and A. Lazarian, Compressible magnetohydrodynamic turbulence: mode coupling, scaling relations, anisotropy, viscosity-damped regime and astrophysical implications, MNRAS 345 (2003) 325 [arXiv:astro-ph/0301062].

[44] A. Beresnyak, A. Lazarian and J. Cho, Density Scaling and Anisotropy in Supersonic Magnetohydrodynamic Turbulence, ApJ 624 (2005) L93 [arXiv: astro-ph/0502547].

[45] B. Burkhart, D. Falceta-Goncalves, G. Kowal and A. Lazarian, Density Studies of MHD Interstellar Turbulence: Statistical Moments, Correlations and Bispectrum, ArXiv e-prints (2008) [0811.0822].

[46] S. Xu, S. Ji and A. Lazarian, On the Formation of Density Filaments in the Turbulent Interstellar Medium, ApJ 878 (2019) 157 [1905.06341].

[47] L.F. Richardson, Atmospheric Diffusion Shown on a Distance-Neighbour Graph, Proceedings of the Royal Society of London Series A 110 (1926) 709.

[48] A. Lazarian, E.T. Vishniac and J. Cho, Magnetic Field Structure and Stochastic Reconnection in a Partially Ionized Gas, ApJ 603 (2004) 180 [physics/0311051]. 
[49] G. Eyink, E. Vishniac, C. Lalescu, H. Aluie, K. Kanov, K. Bürger et al., Flux-freezing breakdown in high-conductivity magnetohydrodynamic turbulence, Nature 497 (2013) 466.

[50] A. Lazarian, G.L. Eyink, A. Jafari, G. Kowal, H. Li, S. Xu et al., 3D turbulent reconnection: Theory, tests, and astrophysical implications, Physics of Plasmas 27 (2020) 012305 [2001.00868].

[51] R. Schlickeiser and J.A. Miller, Quasi-linear Theory of Cosmic-Ray Transport and Acceleration: The Role of Oblique Magnetohydrodynamic Waves and Transit-Time Damping, ApJ 492 (1998) 352.

[52] H.J. Voelk, Cosmic ray propagation in interplanetary space, Reviews of Geophysics and Space Physics 13 (1975) 547.

[53] H. Yan and A. Lazarian, Cosmic-Ray Propagation: Nonlinear Diffusion Parallel and Perpendicular to Mean Magnetic Field, ApJ 673 (2008) 942 [0710.2617].

[54] R.F. Post, Proc. of Second U.N. Int. Conf. on Peaceful Uses of Atomic Energy, Vol. 32, Paper A/Conf. 15/P/377, Geneva, pp. 245-265 (1958).

[55] C.J. Cesarsky and R.M. Kulsrud, Role of Hydromagnetic Waves in Cosmic-Ray Confinement in the Disk. Theory of Behavior in General Wave Spectra, ApJ 185 (1973) 153.

[56] A.U. Abeysekara, A. Albert, R. Alfaro, C. Alvarez, J.D. Álvarez, R. Arceo et al., Extended gamma-ray sources around pulsars constrain the origin of the positron flux at Earth, Science 358 (2017) 911 [1711.06223].

[57] S. Xu, Mirror diffusion of cosmic rays in highly compressible turbulence near supernova remnants, submitted (2021). 